\title{
ENCRUCIJADA EN LA EDUCACIÓN ¿PODREMOS MEJORAR LA PRUEBA PISA EN LOS PAÍSES DE LA REGIÓN?
}

\author{
CROSSROADS IN EDUCATION \\ CAN WE IMPROVE THE PISA TEST \\ IN THE COUNTRIES OF THE REGION?
}

\author{
Marie Lissette Canavesi Rimbaud* \\ mcanavesi@gmail.com
}

Manuel Angel Tomioka Suzuki**

matomioka@hotmail.com
"El problema de la desigualdad en América Latina, no es un problema de crecimiento económico, sino de educación"
Dr. César Gaviria
Presidente de Colombia
1990-1994

\section{RESUMEN}

El programa para la Evaluación Internacional de Alumnos (PISA) de la OCDE, nació con el objetivo de evaluar a los alumnos de aproximadamente 15 años, los mismos que se encuentran casi al finalizar la educación obligatoria, lo que permite medir el grado de conocimiento y habilidades que han obtenido antes de insertarse en la comunidad, frente a los desafíos que ésta plantea. Preocupa la gran diferencia obtenida en el PISA 2012, entre los países del primer mundo y los ocho (8) países latinoamericanos que participaron. Conforme avanzan los años, la diferencia se acrecienta y si no se corrigen de inmediato, ocasionarán mayores desigualdades sociales como está sucediendo actualmente en varios países de América Latina.

El libro Gestión de Sistemas Educativos con Calidad, que demuestra el camino a seguir para obtener la Calidad Educativa, nos guía para enfrentar las próximas evaluaciones PISA 2015, 2018 $\ldots$, al sugerir medidas urgentes e inmediatas que deben ser adoptadas para mejorar los niveles educativos. El crecimiento sostenido que mantenían muchos de los países latinoamericanos en la década anterior (2000-2010) gracias a la exportación de las materias primas y el precio de éstas, está llegando a su fin provocando una desaceleración y deterioro de la economía, y por lo que los gobiernos han dejado de invertir en educación de calidad, en ciencia y tecnología (calidad, productividad e innovación). Una buena educación en matemáticas, ciencia y tecnología hubiera

* Consultora Internacional en el Sistema de Naciones Unidas (1989-2012), Profesora de Posgrados en Universidad de la Empresa (Uruguay), Universidad Mayor de San Andrés, Universidad Militar y Universidad Simón Bolívar (Bolivia), Coordinadora General Académica de la Facultad de Educación, Universidad de la Empresa, UDE (Uruguay).

** Consultor Internacional, Profesor de Posgrados en Universidades Latinoamericanas, Universidad Simón Bolívar (Venezuela), Universidad Mayor de San Andrés (Bolivia), Universidad de la Empresa (Uruguay), Universidad San Martín de Porres (Perú) 
contribuido en diversificar las fuentes de ingresos aportando valor agregado a las materias primas y a sus manufacturas, contando con más ingenieros, científicos y tecnólogos, para desarrollar innovaciones en áreas con ventajas competitivas.

Palabras claves: Calidad, Educación, Evaluación PISA, Gestión, Sistemas Educativos.

\section{ABSTRACT}

The Programme for International Student Assessment (PISA) of the OECD was created with the aim of assessing 15-year-old students, who are about to complete compulsory education. It measures the degree of knowledge and skills they have gained before inserting themselves into the community, to confront the challenges it poses. A matter of concern is the significant difference found in PISA 2012 between first world countries and the eight (8) participating Latin American countries. As years go by, the difference is greater and if this is not corrected immediately, it will cause greater social inequalities and that it is currently the case in several countries in Latin America.

The book Gestión de Sistemas Educativos con Calidad" [Management of Quality Educational Systems], which shows the way forward to attain Educational Quality, gives us some guidance to face the next PISA 2015, 2018 ... assessments, as it suggests urgent and immediate measures that have to be adopted to improve educational levels. The sustained growth that many Latin American countries showed in the previous decade $(2000-2010)$ through the export of raw materials and their price is coming to an end causing a slowdown and the deterioration of the economy. Therefore, governments have stopped investing in quality education, in science and technology (quality, productivity, and innovation). A good education in math, science and technology would have contributed to diversify sources of income giving value added to raw materials and their manufacture, with more engineers, scientists, and technologists, to develop innovations in areas with competitive advantage.

Keywords: Quality, Education, PISA Assessment, Management, Educational Systems

\section{ANTECEDENTES}

En la década de los sesenta del siglo pasado, se dieron las primeras evaluaciones internacionales, por la Asociación Internacional para la Evaluación del Desempeño Educativo (IDEA)'. En la década de los noventa tuvieron lugar las evaluaciones regionales, con el Laboratorio Latinoamericano de Evaluación de la Calidad de la Educación (LLECE) de la UNESCO, y recién en el año 2000 algunos países latinoamericanos iniciaron su participación en la evaluación con el proyecto PISA ${ }^{2}$ (aplicado cada tres años).

De los 61 países que participaron en la última evaluación PISA (mediados del año 2012) desarrollado por la $\mathrm{OCDE}^{3}$, los resultados obtenidos por los ocho (8) países latinoamericanos se encontraban entre los puestos 43 al 61 en Habilidad Lectora y Ciencias y entre el 47 al 61 en Matemáticas, obteniendo las siguientes posiciones: En Matemáticas (que tuvo la mayor incidencia), Chile (47 con 423 puntos), México (49 con 413 puntos), Uruguay (51 con 409 puntos), Costa Rica (52 con 407 puntos), Brasil (54 con 391 puntos), Argentina (55 con 388 puntos), Colombia (58 con 376 puntos) y Perú (61 con 368 puntos). En internet ${ }^{4}$ se muestran los resultados por países con sus respectivos puntajes en cada una de las evaluaciones PISA (matemáticas, ciencia y habilidad lectora) realizadas durante los años 2009 y 2012.

Los primeros ocho (8) puestos (PISA 2012), fueron ocupados (en Matemáticas) por Singapur (1ro. con 573 puntos), Corea del Sur (2do. con 554 puntos), Japón (3ro. Con 536 puntos), Liechtenstein 
(4to. Con 535 puntos), Suiza (5to. con 531 puntos), Países Bajos (6to. con 523 puntos), Estonia (7mo. con 521 puntos) y Finlandia (8vo. con 519 puntos). Las posiciones en Habilidad Lectora y en Ciencias, mantienen aproximadamente el mismo orden.

La evaluación efectuada en el PISA 2009, conservan casi las mismas ubicaciones que las realizadas en el PISA 2012.

Los resultados obtenidos así como la situación de estancamiento de los países latinoamericanos son alarmantes. Los informes que emiten cada año los Ministerios de Educación de los distintos países de América Latina, explican la alta desafiliación, el porcentaje de los repitientes, el bajo porcentaje que alcanzan a las universidades con estudiantes que culminan, así como el alto porcentaje de reprobados durante el primer año universitario y en el primer año de secundaria. También describen el bajo porcentaje de alumnos inscritos en áreas técnicas con relación a las áreas sociales, así como los resultados de las evaluaciones internas y de las evaluaciones PISA.

Pero, lo más inquietante es conocer el impacto de las políticas educacionales que se formulan en cada cambio de gobierno, siendo muy poco o casi nada los resultados que se han obtenido en mejora educacional. Muchas veces, podemos ver que provocan más daño alterando el sistema, porque las medidas que adoptan, solo demuestran desesperación en la búsqueda de cambios rápidos en el corto plazo. Para ello aplican una mayor cantidad de inspecciones, pensando que con estas medidas la educación mejorará.

Lo preocupante es que las medidas que están adoptando los países, pensando en la mejora educacional con apariencia de ser eficientes, resultan que a largo plazo van en contra de la calidad educativa, conforme se demuestra en el libro Gestión de Sistemas Educativos con Calidad5

Es conocido que el proceso de maduración en Educación es lento, con resultados a largo plazo. Basta con calcular que para la evaluación PISA del 2021, los alumnos que pasarán la prueba PISA cursan en estos momentos (2014) el segundo, tercer y cuarto año de Primaria.

La situación en muchísimas escuelas latinoamericanas es muy deficitaria. Por ejemplo en Perú, no existe una computadora por aula, o a veces cuentan solo con un grupo de computadoras antiguas y sin conexión de internet, ni que decir de las infraestructuras (aulas dañadas, baños deteriorados, sin servicio de agua potable ni alcantarillado, sin muebles y sillas adecuadas, sin bibliotecas, sin áreas deportivas, sin comedores, sueldos bajísimos, sin libros ni textos para profesores, carga horaria reducida, etc.). Otro caso sería lo que sucede en Primaria, donde los libros de texto que se entregan a los alumnos en forma gratuita son prestados (debe durar tres años) y solo para poder ser utilizado en horas de clase, prohibiéndose que el estudiante pueda llevarlo a su casa.

Un tema por demás importante, se da con las horas de clases al año que son reducidas si las comparamos con los países del Primer Mundo, o vemos como algunos centros educativos, redujeron las horas para poder compartir las mismas aulas (mañana-tarde) por otro grupo de alumnos.

Para tener una Educación con Calidad, alertamos que es urgente y necesario avanzar rápidamente, porque si no los países de Latinoamérica estarán condenados a un atraso permanente, ya que la ciencia y la tecnología de estos últimos años ha ido creciendo en forma exponencial. Los países desarrollados, generan un gran número de patentes de nuevas invenciones, que representan una de las medidas del avance tecnológico y de la innovación. Por ejemplo todos los países de América Latina y el Caribe, registran 1200 patentes al año (660 de Brasil, 230 de México, 140 de Chile, 80 de Colombia, 26 de Argentina, 18 de Panamá, 13 de Perú, 9 de Cuba y 1 de Venezuela) según la Organización Mundial de la Propiedad Intelectual, (OMPI), y al compararlo con las patentes de los los países desarrollados como Estados Unidos (57000), Japón (44000), China (22000), Alemania (18000), Corea del Sur (12400), vemos la enorme diferencia. 


\section{1. ¿Qué es el PISA? PISA (Programa Internacional de Evaluación de los Alumnos) Evaluación PISA 2009 y 2012}

EI PISAes un estudio periódico y comparativo, promovido, elaborado y organizado por la Organización para la Cooperación y Desarrollo Económico (OCDE), en el cual participan los países miembros y no miembros (asociados) de la organización. El programa Internacional de Evaluación de Estudiantes (PISA por sus siglas en inglés) se aplica cada tres (3) años, cuyo inicio data del año 1997, destinado a la evaluación de estudiantes de 15 años de edad ${ }^{6}$.

El objetivo es evaluar a los alumnos para ver si han adquirido los conocimientos y las habilidades necesarias para la participación plena en la sociedad del saber, verifica cuánto de lo aprendido y asimilado pueden aplicarlo o utilizarlo en situaciones diarias, por lo tanto no evalúan lo que saben (no analiza los programas nacionales), sino lo que pueden hacer con lo que saben.

PISA evalúa competencias en tres áreas: matemáticas, ciencias y lectura, busca conocer en qué medida son relevantes para el bienestar personal, social y económico, midiendo las capacidades de poder entender y resolver problemas, cuyos resultados no solo reflejan la situación de la educación escolar en los países participantes, sino que deberían promover el mejoramiento de la misma.

En cada evaluación, la prueba enfatiza en alguna de las tres áreas antes mencionadas, en el año 2000 el énfasis fue en lectura, en 2003 en matemáticas, en 2006 en ciencias, en 2009 en lectura, en 2012 en matemáticas y en 2015 será en ciencias. Los resultados de las evaluaciones, deberían ser utilizados en la creación de políticas educativas para que los estudiantes adquieran las habilidades y competencias que se requieren en el ámbito nacional como internacional, ya que al conocer los resultados de otros países, se deberían tomar en cuenta las metodologías aplicadas para su éxito o su fracaso, y los factores que explican dichos resultados.

\subsection{Participantes y duración de la Prueba PISA}

$:: 84::$ Los participantes son estudiantes seleccionados a partir de una muestra aleatoria entre las escuelas públicas como privadas del país, elegidos en función de la edad (15 años 3 meses a 16 años 2 meses, al inicio de la evaluación) y no del año o grado escolar en que se encuentren. Primero se elige un determinado número de centros educativos (mínimo de 150 centros educativos por país) y posteriormente se eligen unos 35 alumnos de cada centro educativo. Después de descontar a los alumnos que no participan por diferentes causas (acordadas internacionalmente), la cantidad de alumnos evaluados debe ser mayor de 4500 alumnos en cada país ${ }^{7}$.

El programa PISA busca que los países participantes compitan y establezcan metas cada vez más ambiciosas, en cuanto a su desarrollo educativo. Pisa también compara y analiza las fichas que completan todos los participantes (datos personales, escolares y familiares), así como las fichas que completan los directores de los centros educativos evaluados, cuyos resultados deberían ayudar a los gobiernos a mejorar la formulación de las políticas educativas (hasta el PISA 2012 se ha evaluado a más de un millón de estudiantes).

El tiempo de duración de la prueba es de dos (2) horas, repartido en las tres áreas ya mencionadas, con la prioridad en una de las áreas sobre las otras dos, en cantidad de preguntas y tiempo. En PISA 2012, por ejemplo el énfasis fue en matemáticas con una duración de una hora y veinte minutos, y para las otras dos áreas (ciencia y lectura) de tan solo 20 minutos en cada una, para un total de aproximadamente 55 preguntas, con un formato variado, preguntas de opción múltiple y de respuestas cortas como extensas, habiéndose requerido de solo papel y lápiz. Para la evaluación del 2015, la prioridad será ciencias, además de lectura y matemáticas, se añadirá la resolución de problemas de colaboración y la educación financiera, y por primera vez se utilizarán las computadoras. 
Cuadro No 1 PARTICIPACIÓN DE LOS PAÍSES LATINOAMERICANOS (2000 al 2012)

\begin{tabular}{|c|c|c|c|c|}
\hline PISA 2000 & PISA 2003 & PISA 2006 & PISA 2009 & PISA 2012 \\
\hline 43 países & 41 países & 57 países & 65 países & 65 países \\
\hline \multicolumn{5}{|c|}{ Países de Latino América } \\
\hline Argentina & ------ & Argentina & Argentina & Argentina \\
\hline Brasil & Brasil & Brasil & Brasil & Brasil \\
\hline ----- & ------ & ------ & ------ & Costa Rica \\
\hline Chile & ------ & Chile & Chile & Chile \\
\hline México & México & México & México & México \\
\hline Perú & ------ & ------ & Perú & Perú \\
\hline ----- & ----- & Colombia & Colombia & Colombia \\
\hline ----- & ----- & ------ & Panamá & ----- \\
\hline ----- & Uruguay & Uruguay & Uruguay & Uruguay \\
\hline
\end{tabular}

Fuente: Los autores, con datos de los informes PISA.

Cuadro N 2 PISA 2012: TOTAL DE PAÍSES PARTICIPANTES

\begin{tabular}{|l|l|l|l|}
\hline \multicolumn{2}{|c|}{ PAÍSES OCDE } & \multicolumn{2}{l|}{ Países y Economías Asociadas en PISA 2012 } \\
\hline Alemania & Islandia & Albania & Kazakstán \\
\hline Australia & Israel & Argentina & Letonia \\
\hline Austria & Italia & Brasil & Liechtenstein \\
\hline Bélgica & Japón & Bulgaria & Lituania \\
\hline Canadá & Korea & Chipre & Malasia \\
\hline Chile & Luxemburgo & Colombia & Montenegro \\
\hline Dinamarca & México & Costa Rica & Perú \\
\hline Eslovenia & Nueva Zelanda & Croacia & Qatar \\
\hline España & Noruega & Emiratos Árabes Unidos & Rumania \\
\hline Estados Unidos & Polonia & Federación Rusa & Serbia \\
\hline Estonia & Portugal & China - Hong Kong & Singapur \\
\hline Finlandia & Reino Unido & China - Macao & Tailandia \\
\hline Francia & República Checa & China - Taipei & Túnez \\
\hline Grecia & República Eslovaca & China - Shangai & Uruguay \\
\hline Holanda & Suecia & Indonesia & Viet Nam \\
\hline Hungría & Suiza & Jordania & \\
\hline Irlanda & Turquía & & \\
\hline
\end{tabular}

Fuente: Los autores, con datos del informe PISA 2012. 


\section{3. Áreas de Evaluación del PISA}

\subsubsection{PISA Ciencias}

El conocimiento científico y el uso que se puede hacer de él tanto para identificar preguntas, como para explicar fenómenos científicos nuevos y llegar a conclusiones basadas sobre distintas pruebas, incluye la comprensión de las características de la ciencia como una forma de conocimiento y de la investigación. Tener la percepción de que la ciencia y la tecnología organizan nuestro medio material e intelectual, así como mostrar el deseo de interesarse por cuestiones e ideas relacionadas con la ciencia, y así actuar como ciudadanos reflexivos.

Contenido: Relativos a la física, la química, la biología, la geología y la astronomía.

\subsubsection{PISA Lectura}

Denota la capacidad de un individuo para comprender, utilizar, reflexionar e interesarse por textos escritos, para alcanzar los propios objetivos, desarrollar el conocimiento y participar en la sociedad.

Contenido: Tipo de textos, continuos o de prosa, organizados en oraciones y párrafos (p.ej. narrativos, expositivos, argumentativos, descriptivos, instructivos); textos discontinuos, que presentan la información en forma de listas, gráficos, mapas y diagramas.

\subsubsection{PISA Matemáticas}

Permite identificar la capacidad del individuo para formular, emplear e interpretar las matemáticas en distintos contextos. Incluye el razonamiento matemático y la utilización de conceptos, procedimientos, datos y herramientas matemáticas para describir, explicar y predecir fenómenos. Ayuda a los individuos a reconocer el papel que las matemáticas desempeñan en el mundo, a emitir juicios y

$:: 86::$ decisiones que los ciudadanos reflexivos y comprometidos necesitan.

Contenido: Cuatro áreas: aritmética, álgebra, geometría y estadística, interrelacionadas en forma diversas: cantidad, espacio y forma, cambio y relaciones, incertidumbre y datos.

\subsubsection{PISA Cuestionario}

Los estudiantes que participan deben responder un cuestionario con preguntas sobre si mismos, de sus hogares, de la escuela y de sus experiencias de aprendizaje, con una duración de 30 minutos. Asimismo a los directores de las escuelas participantes se les solicita que completen un cuestionario sobre las características de la escuela y el ambiente del trabajo, con una duración de 30 minutos. En algunos países se distribuyeron cuestionarios a los padres de familia, sobre su participación en la educación de sus hijos, percepción de la escuela, apoyo en el aprendizaje en el hogar, expectativas sobre el futuro de sus hijos y explícitamente relacionado con las matemáticas.

La evaluación de los resultados de aprendizaje, se iniciaron en el año 1960 en los Estados Unidos por la Asociación Internacional para la Evaluación del Desempeño Educativo (IDEA en inglés). En América Latina, algunos países la realizaron y a partir del 2000 se iniciaron en el proyecto PISA (Programe for International Student Assessment) de la Organización para la Cooperación y el Desarrollo Económico (OCDE). Los primeros fueron México y Brasil y posteriormente al 2002, se incorporaron Argentina. Chile, Perú, Uruguay, Colombia, Panamá, República Dominicana y Trinidad y Tobago. Se consideró que podría ser una oportunidad para que los países de la región mejoren la calidad de sus sistemas de evaluación, dirigiéndolo hacia una mejora en la calidad educativa.

En la evaluación del 2012, participaron 65 países con 510.000 estudiantes de 34 países de la OCDE y 31 países asociados, que representaron al $80 \%$ de la población mundial (China, Venezuela, Dubai e India, solo evaluaron algunas ciudades). 


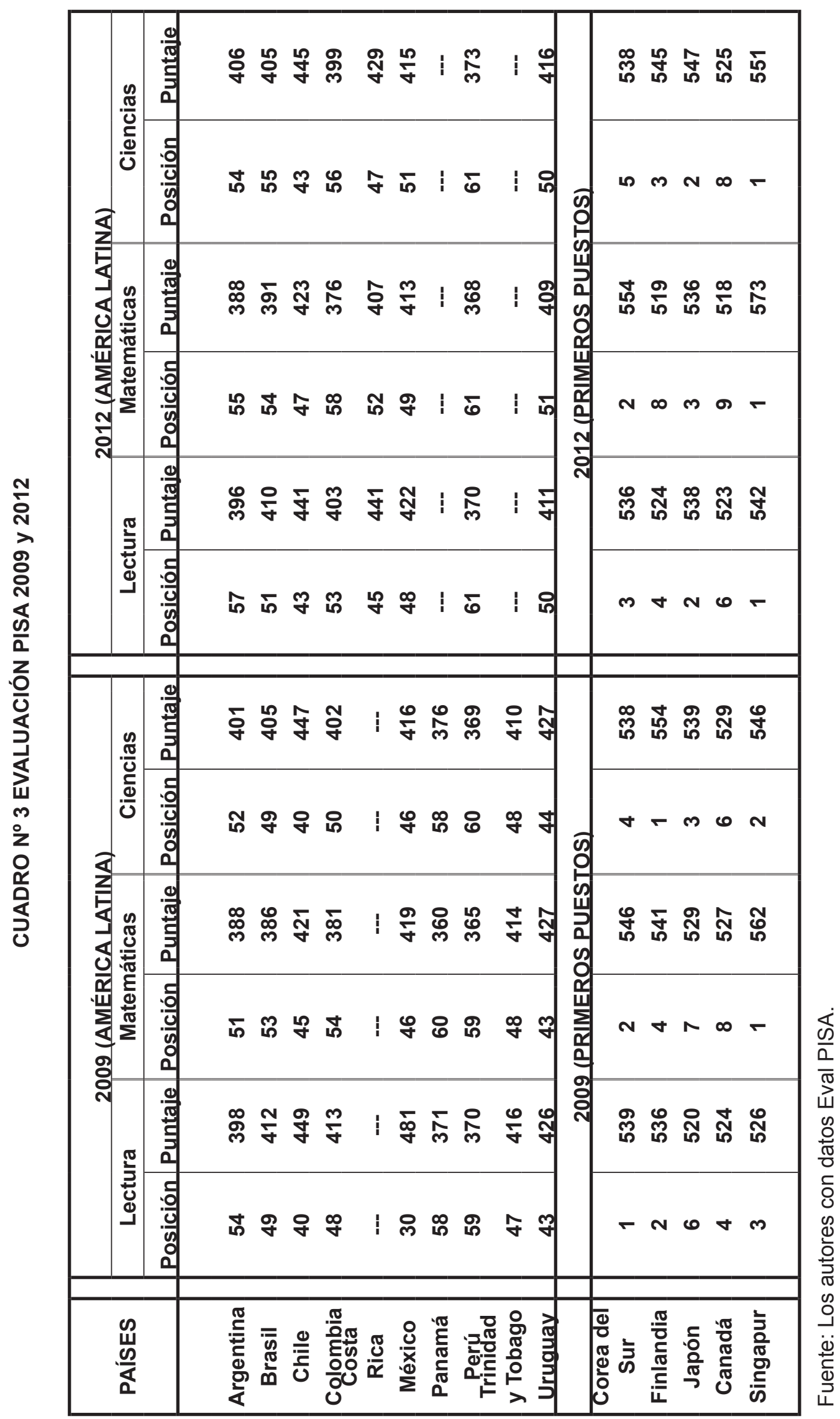




\section{ANÁLISIS DEL RENDIMIENTO PISA 2012, ASOCIADA A LA REALIDAD SOCIOCULTURAL LATINOAMERICANA}

2.1. La evaluación resultante del PISA 2012, señaló amplias diferencias en relación al conocimiento, que divididos en tercios, los países desarrollados ocuparon el primer tercio, mientras que los países latinoamericanos que participaron ocuparon el tercio inferior.

2.2. Los países cuyos centros educativos tienen mayor autonomía respecto a la asignación de recursos, toma de decisiones sobre los currículos, temas de enseñanza, libros de textos a utilizar (para maestros y alumnos), asignaturas a ofrecer, forma de evaluación, etc., obtuvieron mayor rendimiento.

2.3. Los estudiantes que hasta los 15 años, han contado con la ayuda de los padres, en cuanto a seguimiento, ayuda en la comprensión de las lecturas, resolución de ejercicios y problemas de matemáticas, mayor dedicación en tiempo para sus labores escolares y deportivas, e implicarse de manera activa en la educación de sus hijos, aparte de ayudarlos a una mayor formación y seguridad, han logrado obtener mayor puntuación en la evaluación PISA.

2.4. Los alumnos que asistieron a una educación infantil (preescolar, jardín de infancia y guarderías, que se ofrecen para niños a partir de los tres años de edad) de por lo menos un año, han obtenido mayor puntuación que aquellos que no lo hicieron, independiente de su condición económica.

2.5. Los alumnos que pertenecen a escuelas que mantienen una mayor disciplina para favorecer más al aprendizaje, son los que han obtenido mayor puntuación, puesto que los maestros no tienen que invertir tanto tiempo en la creación de un entorno disciplinado, antes de iniciar las tareas de enseñanza. Las interrupciones en clase perturban la concentración de los alumnos y afecta el avance de los mismos. Existen países que prohíben a los maestros imponer mayor disciplina a los alumnos que interrumpen constantemente, con el deterioro del resto de sus compañeros.

2.6. Los alumnos que aprendieron a leer por placer diariamente, obtuvieron mayor rendimiento que los compañeros que no lo hicieron. Los ayuda a ser más eficaces en su aprendizaje y a obtener mayor rendimiento en la escuela, por que la lectura los vincula y los motiva a la investigación, ya sea que la realicen por internet, periódicos, revistas, libros, visitas a las bibliotecas, asistencia a charlas sobre temas específicos, etc., lo que les servirá para su vida profesional.

2.7. Las chicas han tenido mayor desempeño que los chicos en prueba de lectura, en cambio en las pruebas de matemáticas el resultado fue inverso; en ciencias la diferencia ha sido mínima a favor de los chicos.

2.8. Los estudiantes con mayores ventajas socioeconómicas en PISA, han obtenido mayores puntajes, que provienen de escuelas con una mayor proporción de docentes de tiempo completo y grados universitarios avanzados.

2.9. La mayoría de los estudiantes con pobre desempeño en PISA, provienen de entornos socioeconómicos desfavorecidos, existiendo estudiantes que obtuvieron puntajes altos.

2.10. Estudiantes de hijos de inmigrantes (primera generación) o que nacieron en otro país, en promedio obtuvieron puntajes inferiores respecto a compañeros que no tuvieron esos antecedentes.

2.11. Los estudiantes de colegios urbanos obtuvieron mejores niveles de desempeño que los estudiantes de las áreas rurales.

2.12. Los estudiantes provenientes de hogares con solo padre o madre, obtuvieron menor puntaje comparados con estudiantes que provienen de otros tipos de familias.

2.13. Los estudiantes cuyos padres tuvieron una actividad lectora con ellos durante su primer año escolar, obtuvieron mayor nivel de desempeño. 
2.14. Estudiantes que aprendieron a utilizar estrategias para comprender y recordar lo que leen, como subrayar partes importantes del texto o discutir lo que leen con otras personas, tuvieron mejor desempeño en el PISA.

2.15. Los estudiantes que obtuvieron las calificaciones más altas, son aquellos que aprendieron a resumir lo que leen.

2.16. La Institución Educativa, donde se forma el estudiante y ocurre la mayoría de su aprendizaje, tiene un gran impacto en su formación y está influenciado por los recursos (infraestructura física, equipos de enseñanza y ambientes adecuados), materiales de aprendizaje, días y horas al año, maestros a dedicación completa y bien remunerados, y demás aspectos de infraestructura adicionales que requiere una excelente educación.

2.17. Se ha comprobado que las instituciones educativas, donde más estudiantes repiten de año escolar, los resultados han sido inferiores. También se comprueba en donde existen mayores diferencias socioeconómicas, la diferencia de los resultados son mayores.

2.18. Los sistemas escolares exitosos han priorizado la remuneración de los docentes, más que el tamaño del grupo de estudiantes por aula.

\section{MEDIDAS MÍNIMAS NECESARIAS Y URGENTES A IMPLEMENTAR}

Para reducir estas enormes diferencias en los resultados de la evaluación PISA a través de las últimas cinco evaluaciones (2000 al 2012), demostrando que las medidas adoptadas a lo largo de estos años, han sido mínimas o nada productivas, por lo tanto es urgente y necesario implementar medidas en forma inmediata, que estén acordes con planes y objetivos de largo plazo, y nos referimos a 3 áreas, esencialmente en lo que se ofrece en las escuelas:

a) Estudiante: Enseñanza del estudiante, hacia un mejor aprendizaje

b) Docente: Mayor capacitación y preparación de los docentes para una óptima enseñanza

c) Centro Educativo: La Escuela, mejoramiento y equipamiento para una operación en forma más eficiente y eficaz.

Las medidas mínimas necesarias y urgentes a implementar para éstas tres áreas, son:

\subsection{Aplicación de la norma ISO $9001^{8}$ (calidad educativa) normas ISO 9001 (2000) - guía IRAM 30.000}

Las Normas ISO, (Organismo Internacional de Normalización), desde aproximadamente el año 1950 a la fecha, ha venido desarrollando y aprobando, más de 17000 normas que promueven el desarrollo sostenible en todas las áreas, ya sean económicas, ambientales y sociales, con el objetivo fundamental de obtener calidad de los productos o servicios, y por ende de que todos sean iguales o similares (el prefijo "ISO" significa "igual" en español).

Cuando hablamos de implementar ISO 9001 en Educación, nos referimos a redactar todos los procedimientos de todos los procesos internos y externos que se llevan a cabo en el Sistema Educativo de un país.

En referencia a las áreas antes mencionada, las más importantes y urgentes, son:

\subsubsection{Mejorar los libros-textos para los estudiantes de Primaria}

Que sean entregados a los alumnos (no prestados como pasa en algunos países de Latinoamérica), acorde con el libro-texto del maestro 


\subsubsection{Redactar y/o adaptar libros-textos para los maestros de Primaria}

Libros-textos, que existen en todos los países desarrollados y algunos latinoamericanos, y no pretender exigir (todos los días) que los maestros realicen dicha labor, provocando mayores distorsiones en el sistema de educación.

En Educación, los términos "Producto" y "Cliente" suenan extraños, entendiendo por "Producto" al servicio de enseñanza que presta una organización educativa que es la que provee el servicio de enseñanza, y por "Cliente" a los alumnos, padres o tutores, organizaciones que contratan servicios educativos pudiendo ser el Estado Nacional, Departamental, Provincial o Municipal, que reciben el producto. Es posible aplicar estas medidas utilizando la norma ISO 9001, 9002, 9003 y 9004, complementándose con la Norma IRAM 30.000, que es una Guía para la Interpretación, orientación y aplicación de la Norma Iso 9001 en la Educación.

La Norma IRAM 30.000 se puede aplicar a todo tipo de organizaciones educativas, sean públicas o privadas, presencial, semi-presencial o a distancia. Debemos entender que la Norma ISO 9001, no pretende que el Ministerio de Educación, defina que programas educativos debe ofrecer, sino que pueda gestionar los procesos educativos como los administrativos, para asegurar que su producto (servicio de enseñanza), satisfaga las necesidades y expectativas de sus clientes. Ayuda a ordenar, sistematizar e igualar la enseñanza, aumentando el rendimiento operativo, generando ventajas competitivas e igualando en cantidad y profundidad la enseñanza a impartirse.

\subsection{Uniformidad en la metodología de enseñanza (ISO)}

Llamada "Sesión de Aprendizaje" para cada tema o contenido, en donde se menciona el Área, Dominio (¿qué va a hacer?) u Organizador, Capacidad, Indicadores, Instrumento de Evaluación, Bibliografía, Estrategias Metodológicas (¿cómo lo hacemos?) indicando las etapas: inicio, desarrollo (lista de ejercicios y/o lectura para la aplicación de lo aprendido) y cierre (comprobación y metacognición), además de indicar los materiales a utilizar, midiendo el tiempo para cada etapa de la sesión. Este

$:: 90::$ material debe ser entregado a los maestros y no pretender exigir que cada maestro prepare el suyo (más de 5 páginas por tema) con inspecciones punitivas, logrando mayor distorsión en el sistema, sin lograr la uniformidad que se requiere.

\subsection{Capacitación permanente a los maestros}

Si se reparten los libros-textos sugeridos para los maestros; éstos servirán para la auto-capacitación principalmente en la didáctica y el conocimiento del área, teniendo motivo para investigar y complementar lo que considere apropiado.

En los años recientes, los diferentes gobiernos de los países latinoamericanos han logrado aumentar los presupuestos para la Educación debido a los resultados de la prueba PISA, pero sin tener planes específicos a largo plazo, y han pensado que con aumentar las capacitaciones resolverían el problema y están contratando personal y empresas para tal fin.

Los recursos humanos (maestros y profesionales en general) requieren capacitación constante y más en la actualidad, debido a los avances de la ciencia, irán perdiendo competitividad por falta de actualización.

\subsection{Aumento de sueldos a los maestros y cuadruplicar el presupuesto en educación}

En algunos países los sueldos de los maestros son menores que el promedio per cápita del país (muchas veces un lustrabotas gana más) haciendo que la profesión de maestro sea la peor remunerada y con menos incentivo a que los mejores estudiantes deseen postular a dicha profesión. El presupuesto se debe multiplicar varias veces si se quiere crecer y avanzar en producir mejores profesionales, ya sea aumentando los sueldos de los maestros, dotando a las escuelas de las infraestructuras físicas necesarias (aulas, bibliotecas, comedores, baños, áreas deportivas, salas de música, salas de idiomas, mobiliario adecuado, etc.), como de los equipos modernos y materiales necesarios, aumentando las horas y días de enseñanza al año, incrementar la capacitación de 
maestros, libros y textos revisados para los alumnos, libros-textos para los maestros, así como equipamiento continuo de la biblioteca.

En países donde la población infantil se ha incrementado, se han reducido las horas de enseñanza y se comparten las aulas para otros grupos (mañana-tarde), lo que no permite que los alumnos puedan continuar en la escuela a tiempo completo al no poder ampliar las horas de enseñanza.

La evaluación del desempeño de los maestros, debe verse con un programa de formación a largo plazo con su historia de vida, donde se incluye la antigüedad, la calidad de su labor docente, capacitaciones recibidas y aprobadas, cumplimiento de metas, aporte y desempeño al mejoramiento continuo de la organización a la cual pertenece como un equipo.

Los administradores que dirigen las políticas públicas, deben reconocer su propia responsabilidad y comprender que los sistemas creados y perpetuados por ellos ocasionan aproximadamente el $85 \%$ de los problemas y que nada pueden hacer los maestros para solucionarlos o mejorarlos, mientras no exista un cambio en el sistema. Por ejemplo se dió el caso del Presidente Reagan (EE.UU.), que en 1983 pretendió mejorar el rendimiento de las escuelas, indicando que las escuelas públicas estaban fallando y que una forma de mejorarlas sin destinar más fondos federales, era comenzar a pagar a los maestros según sus méritos en vez que por su antigüedad.

Algo similar ocurre en Perú, al entregar un bono a los maestros que consigan que sus alumnos obtengan notas aprobatorias para los exámenes que se aplicarán. Actualmente la administración Obama, al igual que la de Busch, esta premiando a los maestros de escuela en forma individual con mejorar su salario en base a los resultados que sus estudiantes obtengan luego de la aplicación de unos test estandarizados. Esto viola uno de los Principios de Calidad (filosofía Deming) más del $85 \%$ de los resultados de un estudiante son debido al sistema, y el sistema incluye todo lo que rodea al estudiante (aulas, computadores, biblioteca, etc.), como la formación y capacitación de los maestros, auxiliares y personal administrativo, y rompe el orgullo y la satisfacción por el trabajo de los maestros, como factores claves y determinantes de la calidad en cualquier tipo de organización.

\subsection{Especialización por materias como en secundaria}

Para ir adecuándose y mejorando la calidad educativa, a partir del segundo grado de primaria (escuelas con varios salones del mismo grado), debe promoverse la intervención de maestros por especialidad, ya sea en matemáticas, comunicación personal y social (historia, geografía, educación cívica), ciencia y ambiente (biología, física, química), arte y religión, educación física, música, idioma extranjero, etc., logrando que los maestros trabajen en equipo en beneficio de los alumnos.

\subsection{Supervisión adecuada con profesionales formados}

No se puede enviar a supervisar y enseñar a los funcionarios sin una buena preparación que requiere años de especialización y dedicación exclusiva. Si los países aún no han sido capaces de proporcionar buenos libros-textos para los alumnos y para los maestros, menos pueden contar con buenos supervisores, lo que demuestra la complejidad existente.

\subsection{Mayor énfasis en matemáticas, ciencia y tecnología}

Se ha comprobado que se requiere mayor dedicación en esfuerzo y tiempo (horas de clases) hacia las matemáticas, ciencia y tecnología, por la menor elección de carreras profesionales que tienen relación con esas materias. Los alumnos a lo largo de su educación primaria y secundaria, cuando no logran un buen aprendizaje de las matemáticas, sienten ansiedad y tienden a evitarlas, y esos altos niveles de ansiedad tienen consecuencias negativas en el corto plazo, con el menor rendimiento en matemáticas (PISA) y en el largo plazo con la escasez de profesionales que opten por carreras relacionadas con esta materia, principalmente las ingenierías. 


\subsection{Selección de cargos con profesionales preparados, estudios adicionales y años de experiencia}

Existen casos de países que están propiciando exámenes para cargos de director y subdirector de escuelas, sin importar la antigüedad, los años de labor dentro de la institución educativa, sin validar sus antecedentes de trabajo, estudios superiores adicionales, capacitaciones aprobadas, etc., provocando daños que retrasarán la ansiada calidad educativa.

\subsection{Eliminar las supervisiones punitivas}

Con la idea de mejorar la calidad educativa en el corto plazo, se vienen incrementando el número de veces que se realiza la supervisión, lo que crea temor entre los maestros, pretendiendo hacer creer que es la mejor manera de mejorar el proceso de enseñanza. Esta actitud equivocada nace de la desconfianza, de no comprender cómo mejorar el proceso educativo, creando un ambiente de temor, confusión, malestar y disgusto; en cualquier etapa del proceso la inspección no mejora ni incrementa la calidad educativa sin un plan pre-elaborado y/o participativo. Si se continúa con esta política, lo único que se logrará, es administrar para el fracaso.

Los planes de estudio que se fijan y se imponen a los maestros, tienen que ver con las metas arbitrarias, sin haberles entregado el material detallado para tal fin, y se ha hecho costumbre que los maestros la acepten pensando que pueden cubrir los temas asignados en forma eficiente. Las metas son necesarias, pero las que se fijan para los demás sin ofrecer una guía detallada, métodos y medios que lleve a la meta, son contraproducentes, porque generan frustración y resentimiento, por ejemplo, si debe tratar un tema de matemáticas, sin especificar detalle de tiempo, cantidad de ejercicios, tipos de ejercicios, etc., es diferente que un maestro lo desarrolle con dos ejercicios, otro con doce ejercicios. La idea es que se entregue al maestro el material correspondiente a la didáctica, y los ejercicios tipos y mínimos a realizarse en clase, incluyendo el tiempo.

\subsection{Eliminar los exámenes para clasificar a los maestros}

$:: 92::$

Existen países que debido a que las remuneraciones de los maestros son tan bajas, independiente de los años de experiencia (por ejemplo, en el caso del Perú, el 80\% percibe el salario mínimo), en una escala de 1 al 5 , la mayoría la colocaron en el 1, y que para aspirar aumentos salariales deberán aprobar exámenes (uno o dos veces por año, con un cupo por cada nivel).

Este método que va en contra de toda filosofía de calidad, ha roto toda capacidad de trabajo en equipo, y de ejercer su profesión con orgullo, al no acreditar su antigüedad, experiencia, estudios y capacitaciones realizadas a lo largo de su vida como docente, aumentando la desconfianza. Sólo se está administrando a base de números y cuotas, haciendo culpables a los maestros por los resultados obtenidos (PISA) y resultados que se llevan a cabo en forma interna en cada país, por problemas que están fuera del control de los maestros, creando un ambiente de temor, premiando y castigando debido a las variaciones que han sido originadas por la administración central.

\subsection{Modificar las reglas sobre la disciplina escolar}

En los últimos años el deterioro de la disciplina en los centros educativos es muy grande, en cuanto a que la autoridad del maestro se ha visto menoscabada, hasta el punto de recibir amenazas de los padres de familia y hasta de los mismos alumnos, sin que exista autoridad que pueda regularla, por las normas impuestas por el mismo Ministerio de Educación, en cuanto a mantener la disciplina dentro del aula.

Se pueden citar algunas de ellas: no poder retirar al alumno del aula que entorpece el trabajo por su mal comportamiento (ruido) y/o por generar conflictos (verbales y físicos) dentro del aula, o que los alumnos que llegan con tardanza pueden ingresar en cualquier momento interrumpiendo la sesión de clases, alterando el orden de la misma. Estos hechos deterioran enormemente el aprendizaje como ocasionan pérdidas de horas efectivas de clases. 


\subsection{Mejora de la infraestructura física, materiales y equipos de enseñanza apropiados}

Nos referimos a las mejoras, ampliaciones, remodelaciones y/o refacciones de las infraestructuras físicas de las escuelas (aulas, mobiliario de alumnos y maestros, baños, áreas deportivas, comedores, bibliotecas, áreas administrativas, salas de maestros, bibliotecas, computadores, etc.), así como establecer la vigilancia y mantenimiento respectivos.

\subsection{Utilización de la curva normal ${ }^{10}$, para la mejora contínua}

Todos actos, hechos, situaciones, acciones, conductas, actuaciones, comportamientos, de los diferentes los procesos y fenómenos naturales, psicológicos, antropológicos, económicos, ambientales, culturales, deportivos, musicales, de entretenimiento, etc., se pueden registrar y medir a través de la Distribución Normal o Campana de Gauss, por lo tanto los fenómenos o sucesos que se viven en Educación y fuera de ella, están comprendidos dentro de esta curva Normal, como las notas de estudiantes, asistencia a clases, tardanzas, inasistencias, horario de entrada, cumplimiento de tareas, salarios de maestros y personal administrativo, así como participación en actividades, comportamiento, participación de los padres de familia, soporte de la administración central, enfermedades de los alumnos y maestros, trabajos del personal administrativo, funcionamiento de cualquier área de apoyo (biblioteca, comedor, baños, vigilancia, limpieza, etc.)

La utilización básicamente es para determinar si las medidas efectuadas de un proceso, suceso o hecho, están todas dentro de la curva normal o fuera de ella, lo que nos determinará si pertenecen a variaciones comunes (dentro de la curva) o a variaciones especiales (fuera de la curva). Para su utilización es necesario calcular la media y la desviación estándar de los datos que se recopilan.

Para lograr la calidad educativa, debe entenderse y medirse con ayuda de la estadística, y entender que en todo proceso existen dos tipos de variación: la especial y la común. La variación especial se debe a una causa específica y es atribuible a la administración central por no disponer de políticas, normas y procedimientos (ISO), infraestructura física, equipamiento en la escuela para la buena marcha de la misma, que por lo general causan del $10 \%$ al $20 \%$ de los problemas; la variación común existe en todo el proceso, genera altos costos, baja calidad y baja productividad que representan entre el $80 \%$ al $90 \%$ de las variaciones.

La mayoría de las veces, sin analizar a que tipo de variación es debida, se toman medidas para solucionar un problema, corriendo el riesgo de crear más variación en el sistema y más problemas que vendrán posteriormente. Si se detectan que son variaciones especiales, los maestros no pueden hacer nada para modificarlas o solucionarlas, siendo la administración central la que debe reconocerlas, ya que es debido a ellos y corregirlos sin culpar a nadie, sino a sí mismos. Si son variaciones comunes, como su nombre lo dice se dan normalmente de acuerdo al sistema implantado (Curva Normal), por lo que tampoco se puede atribuir a los maestros, pero si se puede reducir esta variación, ya que ésta se debe al sistema implantado y que afecta a toda la organización y es perfectible a una mejora continua por la administración central. Es importante conocer que se debe proceder a la mejora de las variaciones comunes, siempre y cuando se hayan eliminado todas las variaciones especiales y dejar que el sistema se pueda llevar a control estadístico y se estabilice. Las variaciones especiales generalmente solo se pueden detectar con la ayuda de técnicas estadísticas adecuadas, ejecutadas por personas competentes que posean conocimientos profundos de estadística.

El desconocimiento de estas reglas básicas para lograr la calidad, de muchas administraciones educativas al no comprender ni cómo distinguir entre estas dos variaciones, culpan a los maestros y personal administrativo. El personal educativo es impotente ante las variaciones comunes, ya que éstas tratan de problemas del sistema y el sistema lo implanta y maneja la administración, los maestros se limitan a laborar en el sistema impuesto (ubicación, local físico, políticas, normas y procedimientos vigentes, horarios, sueldos, transporte, etc.) y es poco lo que puede hacer el maestro con locales poco apropiados, con mínimas facilidades de equipamiento, deficiencias en el mantenimiento, fallas en la iluminación, bibliotecas insuficientes, baños en mal estado, falta de agua y alcantarillado, etc. 


\section{CONCLUSIONES Y RECOMENDACIONES}

\section{La gestión en los sistemas educativos, no es diferente a la gestión de otros tipos de organizaciones.}

$\diamond \quad$ Recordar que el estudiante no es el producto, el "producto" es la educación del estudiante o el servicio de enseñanza que presta una organización educativa. Por eso es importante que el estudiante sea un participante activo en el diseño del producto, ya que es la persona que más tiempo permanece en el sistema educativo.

$\diamond \quad$ “Organización Educativa" es la organización que provee el servicio de enseñanza.

$\diamond \quad$ Podemos llamar "Clientes" a la organización o persona que recibe el producto (alumnos, padres o tutores y organismos que contratan servicios educativos).

$\diamond \quad$ Es vital y de la mayor importancia la mejora de la calidad educativa, ya que el estudiante vivirá con el producto recibido (la educación) por el resto de su vida, igualmente los padres y la familia que pagan por el producto, vivirán y compartirán con el mismo. Los empleadores dependerán de la educación del graduado para alcanzar los objetivos de su empresa, y la sociedad en general espera un ciudadano económicamente activo y contribuyente. Los errores y fracasos que se produzcan en la educación, tendrán un alto costo de oportunidad en la sociedad.

$\diamond \quad$ Es de hacer notar que normalmente se utilizan índices para medir los rendimientos y evaluaciones que se practican, obtenidos de otros países o los obtenidos de escuelas líderes del país ("benchmarking") para utilizarlos como metas pensando que es lo correcto y adecuado. Antes deben analizarse las condiciones socioeconómicas, políticas, geográficas, ambientales, culturales, alimenticias, etc., que nos servirán para entender y conocer qué valores deben fijarse como metas y qué deben ir modificándose.

$:: 94::$

$\diamond \quad$ Se debe eliminar una situación que se repite y se considera óptima, que los maestros instruyan e inspeccionen a otros, sin la preparación adecuada, y posteriormente la cadena continúa, lo cual representa un deterioro sin límite, por eso se debe instituir la formación formal y capacitación continua con personal altamente preparado.

$\diamond \quad$ Se debe ir eliminando las medidas enunciadas que se practican en la actualidad, por la acreditación. Estas disposiciones aparentan ser efectivas y hasta reciben la aprobación de la colectividad, con un gasto enorme del presupuesto. Entre las que se encuentran: la forma de aumentar los salarios a los maestros por medio de exámenes, o la selección a los directores y subdirectores a través de un examen único, el aumento progresivo de las inspecciones punitivas a los maestros (mediante un sistema de notas), $u$ otorgar bonos a los maestros cuyos alumnos aprueben ciertas pruebas, o intentar que los mismos maestros con algunas horas de instrucción sirvan de capacitadores a sus colegas, inversiones sin objetivos a largo plazo al no saber detectar los problemas debido a las variaciones tanto especiales como comunes, etc. Son medidas de corto plazo que producen deterioro a largo plazo en contra de la calidad educativa, con un gran aumento de alteración en los cuadros clínicos por el stress y la depresión en los maestros. 


\section{ÍNDICE DE FUENTES}

\section{SECUNDARIAS}

\section{Bibliografia}

Deming, W. Edwards (1989): Calidad, Productividad y Competitividad, la salida de la crisis. Madrid, Ed. Díaz de Santos S.A.

Eduteka (2009): Informe PISA (2009) http://www.eduteka.org/Pisa2009.php -(2012) Informe PISA (2012)- http://www.eduteka.org/Pisa2012.php

Gitlow, Howard S., Gitlow Shelly J. (1987): Cómo Mejorar la Calidad y la Productividad con el Método Deming, Bogotá, Editorial Norma

González, S. (1998): ISO 9000: Implantación de Sistemas de Calidad. Valencia, Vadell Hermanos Editores, C.A.

Informe McKinsey \& Company: (9/2007) Ocho claves para pasar de un sistema educativo de bajo desempeño a uno bueno USA, Michael Barber y Mona Mourshed

IRAM (Instituto Argentino de Normalización y Certificación) 30.000 (s/f): Guía de la Interpretación de la Norma 9001:2000 para la Educación,: http://codece.edu.ar/docs/IRAM.pdf , http://www.iram30000 ISO (s/f) International Organization for Standardization, - http://www.iso.ch , ISO $9001 \mathrm{http}: / / \mathrm{www}$.isotools.org/normas/calidad/iso-9001

OECD (s/f) Organización para la Cooperación y el Desarrollo Económico. http:// www.oecd.org/pisa/ pisaenespaol.htm

Oppenheimer, Andrés (2005): Cuentos Chinos, Buenos Aires, Editorial Sudamericana S.A.

Oppenheimer, Andrés (2010): ¡Basta de Historias!, Buenos Aires, Editorial Sudamericana S.A.

Oppenheimer, Andrés (2014): ¡Crear o Morir! La esperanza de Latinoamerica y las cinco claves de la innovación, Penguin Random House Grupo Editorial, S.A., de C.V., México, D.F.

Tomioka M., Quijano A., Canavesi L. (2014): Gestión de Sistemas Educativos con Calidad, USA, Editorial Lulu

Recibido el 28 de octubre de 2014 Aceptado el 09 de diciembre de 2014 